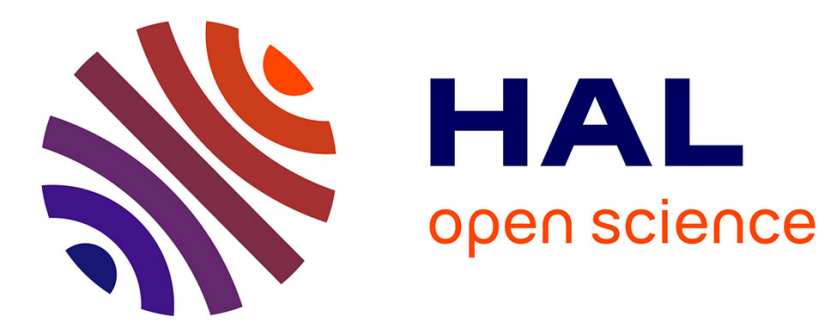

\title{
A multiscale approach to model the mechanical behaviour of heterogeneous hyperelastic membranes
}

Luc Meunier, Laurent Orgéas, Grégory Chagnon, Denis Favier

\section{To cite this version:}

Luc Meunier, Laurent Orgéas, Grégory Chagnon, Denis Favier. A multiscale approach to model the mechanical behaviour of heterogeneous hyperelastic membranes. Sixth European conference on constitutive models for rubber, Sep 2009, Dresde, Germany. pp.429-434. hal-01395961

\section{HAL Id: hal-01395961 \\ https://hal.science/hal-01395961}

Submitted on 13 Nov 2016

HAL is a multi-disciplinary open access archive for the deposit and dissemination of scientific research documents, whether they are published or not. The documents may come from teaching and research institutions in France or abroad, or from public or private research centers.
L'archive ouverte pluridisciplinaire HAL, est destinée au dépôt et à la diffusion de documents scientifiques de niveau recherche, publiés ou non, émanant des établissements d'enseignement et de recherche français ou étrangers, des laboratoires publics ou privés. 


\title{
A multiscale approach to model the mechanical behaviour of heterogeneous hyperelastic membranes
}

\author{
L. Meunier, L. Orgéas, G. Chagnon \& D. Favier \\ In memory of Luc Meunier
}

CNRS/Université de Grenoble, Laboratoire Sols-Solides-Structures-Risques (3S-R), Grenoble Cedex, France

\begin{abstract}
Within the framework given by the homogenisation method with multiple scale asymptotic expansions, a theoretical and numerical analysis is proposed in order to study the mesoscale behaviour of hyperelastic thin membranes exhibiting geometrical or material heterogeneities at the microscale. Results deduced from this multiscale approach are then used to analyse the mechanical behaviour of a crenellated thin membrane made up of a compressible Neo-Hookean material.
\end{abstract}

\section{INTRODUCTION}

Many structures and materials such as thin heterogeneous and/or fibre-reinforced elastomers structures, thin living tissues, manufactured woven or non-woven $2 \mathrm{D}$ textiles, behave as a first and reasonable approximation as elastic and anisotropic membranes undergoing finite strains.

In this work, we propose to estimate, from a theoretical and numerical multiscale approach, the mesoscale effective mechanical properties of such membranes, (i) starting from the description of both the microstructure and the mechanical equilibrium of the membranes at the microscale, and (ii) assuming that the involved materials at the microscale behave as hyperelastic bodies.

Firstly, the above micromechanical problem at the heterogeneity scale is upscaled by using the homogenisation method with multiple scale asymptotic expansions for periodic structures (Bensoussan et al. 1978; Sanchez-Palencia 1980; Caillerie 1984; Auriault 1991; Pruchnicki 1998). The homogenisation also provides suitable localisation problems to be solved within the Representative Elementary Volumes (REV) in order to compute the effective hyperelastic responses of the membranes.

Secondly, these boundary values problems are implemented into a Finite Elements software and the effective mesoscale properties of heterogeneous membranes are investigated quantitatively. The considered membrane is rather simple: it is made up of hyperelastic materials and it exhibits crenellated cross sections. Hence, its associated $\mathrm{REV}$ is subjected to meso-scopic in plane loadings (uniaxial/biaxial tensions, pure shear) and their resulting mesoscopic behaviours (stress levels, evolving anisotropy) are analysed and discussed.

\section{PROBLEM STATEMENT}

We consider a thin heterogeneous plate, e.g. that shown in figure 1(a), with an initial average thickness $e_{0}$ along the $\underline{\mathbf{e}}_{3}$ direction and with a characteristic in-plane initial macroscopic dimension $L_{0}$ (in $\left.\left(\underline{\mathbf{e}}_{1}, \underline{\mathbf{e}}_{2}\right)\right)$. The plate is made up of a periodic assembly of Representative Elementary Volumes (REV) with a characteristic in-plane initial dimension $l_{0}$. It is supposed that the thickness $e_{0}$ and the REV in-plane length $l_{0}$ are of the same order of magnitude, i.e $e_{0} / l_{0} \approx \mathcal{O}(1)$, and that they are very small with respect to the in-plane size $L_{0}$ of the plate, i.e $e_{0} / L_{0} \approx l_{0} / L_{0} \approx \mathcal{O}(\varepsilon)$, the scale separation parameter $\varepsilon$ being very small. A typical example of such REV's is shown in figure 1(b).

For a sake of simplicity, we assume here that the plate is only subjected to in quasi-static and in-plane mechanical loadings without volumetric forces. With the example shown in figure 1(b), this means that the upper $\Gamma^{+}$and lower $\Gamma^{-}$surfaces of the REV are not loaded.

By noting respectively $\underline{\mathbf{X}}$ and $\underline{\mathbf{X}}$ the initial and current position vectors of materials points and $\underline{\mathbf{u}}(\underline{\mathbf{X}})=\underline{\mathbf{X}}-\underline{\mathbf{X}}$ the displacement vector, the first momentum balance equation corresponding to this problem and to be solved in the initial configuration is:

$\underline{\operatorname{Div}}_{X} \underset{\pi}{=} \underline{\mathbf{0}}$,

where $\operatorname{Div}_{X}$ is the divergence operator with respect to the initial configuration and position vectors $\underline{\mathbf{X}}$. 


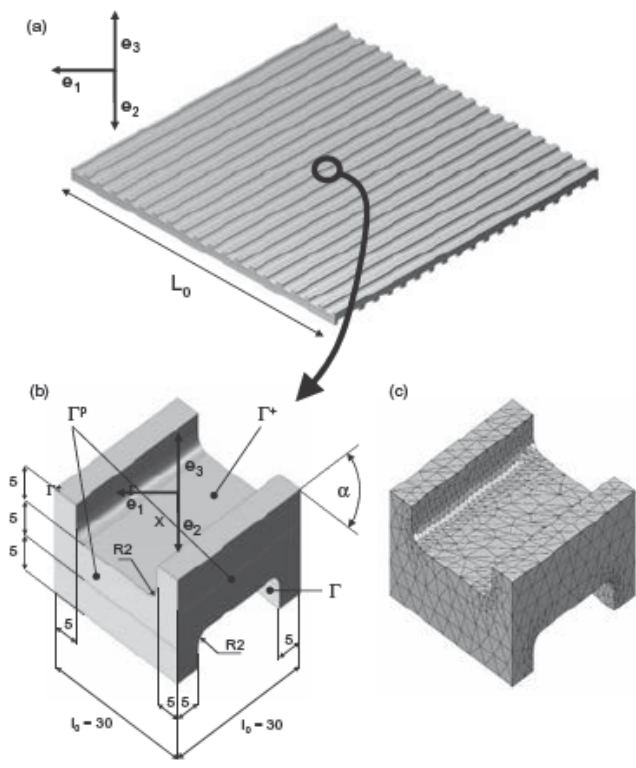

Figure 1. Typical plate which is here considered (a), geometry with $\alpha=65^{\circ}$ (b) and P2 tetrahedral FE mesh ( $\approx 50000$ degrees of freedom) (c) of the Representative Elementary Volume of the considered membrane (dimensions in $\mathrm{mm}$ ).

From the second momentum balance equation, the first Piola-Kirchhoff stress tensor $\stackrel{\pi}{=}$ must verify:

$$
\underline{\pi} \cdot \underline{\underline{\mathbf{F}^{t}}}=\underline{\underline{\mathbf{F}}} \cdot \boldsymbol{\pi}^{t}
$$

In the last equation, $\underline{\mathbf{F}}$ is the transformation gradient defined as:

$\underline{\underline{\mathbf{F}}}=\underline{\underline{\delta}}+\underline{\underline{\mathbf{G r a d}}}_{X}^{\underline{\underline{\mathbf{u}}}}$

$\stackrel{\delta}{=}$ and $\underline{\underline{\text { Grad }}} X$ being the identity tensor and the gradient operator with respect to $\underline{\mathbf{X}}$, respectively.

Besides, the plate is supposed to be made up of compressible hyperelastic materials, the characteristic stiffness of which being of the same order of magnitude. Their stress tensors $\underset{\boldsymbol{\pi}}{\boldsymbol{n}}$ can therefore be written as:

$\stackrel{\pi}{=}=\frac{\partial W(\underline{\underline{\mathbf{F}}})}{\partial \underline{\mathbf{F}}}$,

The local strain energy function $W$ per unit of unde-formed volume is supposed to be positive and such that $W(\delta)=0$. For example, let us assume that the membrane is made up of compressible
Neo-Hookean materials. In this situation, $W$ is expressed as:

$$
W=\frac{1}{2}\left(\mu\left(I_{1} J^{-\frac{2}{3}}-3\right)+k(1-J)\right),
$$

where

$$
J=\operatorname{Det} \underline{\underline{\mathbf{F}},} I_{1}=\operatorname{Tr}\left(\underline{\underline{\mathbf{F}}}^{t} \cdot \underline{\mathbf{F}}\right)
$$

and where $\mu(\mathbf{X})$ and $k(\mathbf{X})$ are the shear modulus and the compressibility modulus, respectively.

\section{UPSCALING}

\subsection{Introduction}

In order to obtain the equivalent mesoscopic mechanical behaviour of the as-described problem, the homogenisation method with multiple scale asymptotic expansions is now used (Bensoussan et al. 1978; Sanchez-Palencia 1980; Caillerie 1984; Auriault 1991). Hence, provided a good scale separation between $L_{0}$ and $l_{0}$, the problem can be tackled by introducing two distinct and independent dimensionless space variables, i.e. $\underline{\tilde{Y}}$ and $\underline{\mathbf{Z}}$ (the symbol " " " denotes quantities which are defined in $\left.\left(\underline{\mathbf{e}}_{1}, \underline{\mathbf{e}}_{2}\right)\right)$ The mesoscopic in-plane space variable $\underline{\tilde{\mathbf{Y}}}=\underline{\tilde{\mathbf{X}}} / L_{0}$ is defined in the $\left(\underline{\mathbf{e}}_{1}, \underline{\mathbf{e}}_{2}\right)$ plane $\left(Y_{3}=\overline{0}\right)$ and characterises the membrane geometry at the mesoscopic scale. The microscopic one, i.e. $\underline{\mathbf{Z}}=\underline{\mathbf{X}} / l_{0}$, characterises the membrane geometry in the REV's. Thus, any scalar function $\varphi(\underline{\mathbf{X}})$ is now written as a function of $\underline{\tilde{\mathbf{Y}}}$ and $\underline{\mathbf{Z}}$, i.e. $\varphi(\underline{\tilde{\mathbf{Y}}}, \underline{\mathbf{Z}})$. Therefrom, it is further assumed that the displacement field $\underline{\mathbf{u}}(\underline{\tilde{\mathbf{Y}}}, \underline{\mathbf{Z}})$ can be expressed as an asymptotic expansion in powers of $\varepsilon$ :

$$
\underline{\mathbf{u}}=\underline{\mathbf{u}}^{(0)}(\underline{\tilde{\mathbf{Y}}}, \underline{\mathbf{Z}})+\varepsilon \underline{\mathbf{u}}^{(1)}(\underline{\tilde{\mathbf{Y}}}, \underline{\mathbf{Z}})+\varepsilon^{2} \underline{\mathbf{u}}^{(2)}(\underline{\tilde{\mathbf{Y}}}, \underline{\mathbf{Z}})+\ldots
$$

where the displacement fields $\underline{\mathbf{u}}^{(i)}$ are supposed to be $\underline{\mathbf{Z}}$-periodic on the lateral surface $\Gamma^{p}$ of the REV (see figure 1(b)).

Accounting for this last assumption, the homogenisation method consists (i) in introducing the new set of space variables $\underline{\underline{Y}}$ and $\underline{Z}$ together with the above asymptotic expansion (7) in the problem (1-6) and (ii) in identifying and solving the problems arising at the different $\varepsilon$-orders.

\subsection{Main results}

Theoretical developments are very similar to what was achieved by Pruchnicki (Pruchnicki 1998) for Saint Venant-Kirchhoff materials. We have here extended his work to a larger class of 
hyperelastic materials and we have also avoided any a priori assumption on the first order displacement field $\underline{\mathbf{u}}^{(0)}$. The reader is referred to his work (Pruchnicki 1998) and to that of Meunier (Meunier 2009) for details about the theoretical developments. We briefly summarise hereafter the main results deduced from the approach:

- From the assumptions stated for $W$, it can be shown that the first order displacement field only depends on the in-plane mesoscopic space variable, i.e. $\underline{\mathbf{u}}^{(0)}=\underline{\mathbf{u}}^{(0)}(\underline{\tilde{\mathbf{Y}}})$.

- The mesoscopic equivalent continuum is a $2 \mathrm{D}$ membrane. In the $\left(\underline{\mathbf{e}}_{1}, \underline{\mathbf{e}}_{2}\right)$ plane, its first momentum balance equation in the initial configuration is expressed as:

$\underline{\mathrm{Div}}_{\tilde{X}}\left\langle\underline{\underline{\tilde{\pi}}}^{(0)}\right\rangle=\underline{\tilde{\mathbf{0}}}$,

where the mesoscopic first order Piola-Kirchhoff stress tensor $\left\langle\underline{\underline{\tilde{\pi}^{(0)}}}\right\rangle$ is defined as

$\left\langle\underline{\underline{\pi}}^{(0)}\right\rangle=\frac{1}{V_{0}} \int_{\Omega_{0}} \stackrel{\tilde{\pi}^{(0)}}{=} \mathrm{d} V$,

such a volume averaging being performed on the initial solid domain $\Omega_{0}$ of the REV, of initial volume $V_{0}$. The local first order stress tensor $\tilde{\pi}^{(0)}$ involved in the last equation is defined from:

$\underline{\pi}^{(0)}=\frac{\partial W\left(\underline{\underline{\tilde{\mathbf{F}}}}^{(0)}+\underline{\underline{\mathbf{H}}}^{(1)}\right)}{\partial\left(\underline{\underline{\mathbf{F}}}^{(0)}+\underline{\underline{\mathbf{H}}}^{(1)}\right)}$,

where

$\underline{\underline{\tilde{\mathbf{F}}}}^{(0)}=\underline{\underline{\tilde{\delta}}}+\underline{\underline{\mathbf{G}}}_{\underline{\mathbf{r a d}}}^{\tilde{X}} \underline{\tilde{\mathbf{u}}}^{(0)}$

and

$\underline{\underline{\mathbf{H}}}^{(1)}=\underline{\underline{\operatorname{Grad}}}_{X} \varepsilon \underline{\mathbf{u}}^{(1)}$

From (2), it also follows that $\left\langle\underline{\underline{\tilde{\pi}^{(0)}}}\right\rangle$ verifies:

$\left\langle\underline{\underline{\tilde{\pi}}}^{(0)}\right\rangle \cdot \underline{\underline{\tilde{\mathbf{F}}}}^{(0)^{t}}=\underline{\underline{\mathbf{F}}}^{(0)} \cdot\left\langle\underline{\underline{\tilde{\pi}}}^{(0)}\right\rangle^{t} \cdot$

- The mesoscopic first order Piola-Kirchhoff stress tensor $\left\langle\underline{\underline{\tilde{\pi}}}^{(0)}\right\rangle$ is defined as the partial derivative of the mesoscopic strain energy function $\left\langle W\left(\underline{\tilde{\mathbf{F}}}^{(0)}\right.\right.$, microstructure $\left.)\right\rangle$ with respect to the first order $2 \mathrm{D}$ transformation gradient $\tilde{\underline{\mathbf{F}}}^{(0)}$ :

$$
\langle\underline{\underline{\pi}}(0)\rangle=\frac{\partial\left\langle W\left(\underline{\tilde{\mathbf{F}}}^{(0)}, \text { microstructure }\right)\right\rangle}{\partial \underline{\tilde{\mathbf{F}}}^{(0)}},
$$

- The first order stress tensor $\left\langle\underline{\tilde{\pi}}^{(0)}\right\rangle$ can be obtained by solving in the REV the following boundary value problem:

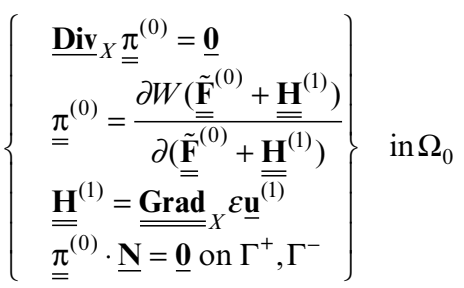

where the first order periodic fluctuation of the displacement field $\mathcal{\varepsilon} \mathbf{u}^{(1)}$ is the unknown field, $\mathbf{N}$ is the unit vector normal to the external surfaces $\Gamma^{+}$and $\Gamma^{-}$, and where $\tilde{\mathbf{F}}^{(0)}$ is given and constant within the entire REV.

\section{APPLICATION TO A SIMPLE MEMBRANE}

By following the previous theoretical framework, it is now possible to compute the effective properties of hyperelastic membranes.

\subsection{Considered membrane geometry and material}

For that purpose, we consider a thin plate made up of upper and lower crenellated profiles. It is shown in figure 1(a). The corresponding REV and its dimensions are given in figure 1(b). As shown from this figure, the upper and lower crenellated profiles are identical but they are not parallel: they respectively make an angle $\pm \alpha / 2= \pm 32.5^{\circ}$ with respect to the $\underline{\mathbf{e}}_{1}$ direction.

For the sake of simplicity, the membrane is assumed to be made up of a unique material, the mechanical behaviour of which is defined from the compressible Neo-Hookean hyperelastic strain energy function (5). The constitutive parameters associated with this function, i.e. $\mu$ and $k$, are assumed to be constant in the whole REV, they were set to 1 and $1000 \mathrm{MPa}$, respectively. Hence, the considered material can be regarded as quasi-incompressible.

In order to study the mesoscopic mechanical behaviour of such an heterogeneous membrane, i.e. to analyse the relationship between $\langle\underline{\underline{\underline{\pi}}}(0)\rangle, \underline{\tilde{\mathbf{F}}}^{(0)}$ and the membrane microstructure (see (14)), the 
boundtary values problem (15) was solved on the considered REV for given values of the $2 \mathrm{D}$ mesoscopic transformation gradient $\tilde{\mathbf{F}}^{(0)}$. This allows to estimate the displacement fluctuation $\varepsilon \underline{\mathbf{u}}^{(0)}$, then the local stress tensor $\tilde{\pi}^{(0)}$ (from (15b), and finally the mesoscopic stress tê̄nor $\left\langle\tilde{\pi}^{(0)}\right\rangle$ (from (9)).

For that purpose, the weak form of this problem was implemented into the Finite Elements (FE) code Comsol Multiphysics, and the REV was meshed using FE elements with second order polynomial form functions (P2, see figure 1(c)). Such a highly nonlinear formulation was solved incrementally, with an iterative Newton's like algorithm at each time step.

\subsection{Anisotropy}

A first type of numerical experiments was achieved in order to analyse the mechanical anisotropy of the considered membrane. Hence, the REV was subjected to a plane strain tension along the $\underline{\mathbf{e}}_{1}$ direction:

$$
\underline{\tilde{\mathbf{F}}}^{(0)}=\lambda_{11} \underline{\mathbf{e}}_{1} \otimes \underline{\mathbf{e}}_{1}
$$

up to an axial elongation $\lambda_{11}=2$. Then, the REV was rotated by an angle $\theta$ in the $\left(\underline{\mathbf{e}}_{1}, \underline{\mathbf{e}}_{2}\right)$ plane, and the same numerical experiment was achieved. This was repeated for various values of $\theta$ ranging from $0^{\circ}$ to $180^{\circ}$. Therefrom, the dimensionless stresses components:

$$
\left\langle\tilde{\pi}_{11}^{(0)}\right\rangle^{*}=\frac{\left\langle\tilde{\pi}_{11}^{(0)}\right\rangle(\theta)}{\left\langle\tilde{\pi}_{11}^{(0)}\right\rangle(\theta=0)} \text { and }\left\langle\tilde{\pi}_{22}^{(0)}\right\rangle^{*}=\frac{\left\langle\tilde{\pi}_{22}^{(0)}\right\rangle(\theta)}{\left\langle\tilde{\pi}_{22}^{(0)}\right\rangle(\theta=0)}
$$

were built and analysed as functions of the orientation angle $\theta$. This is illustrated in figure 2, in which these dimensionless stress ratios were plotted as functions of $\theta$, for two values of the imposed mesoscopic elongation $\lambda_{11}$ :

- Even if the membrane is made up of a unique material, the architecture of the REV induces noticeable anisotropy: stresses components depend on $\theta$. For example the dimensionless stress component $\left\langle\tilde{\pi}_{11}^{(0)}\right\rangle^{*}$ is approximately $25 \%$ higher when $\theta=90^{\circ}$ than when $\theta=0^{\circ}$. This trend is reversed but less pronounced for the second dimensionless stress component $\left\langle\tilde{\pi}_{22}^{(0)}\right\rangle^{*}$.

- The anisotropy magnitude evolves as the imposed mesoscopic elongation is increased. For example, it is approximately twice higher for $\left\langle\tilde{\pi}_{22}^{(0)}\right\rangle^{*}$ when $\lambda_{11}$ goes from 1.25 to 2 . This is much less emphasised and this trend is reversed for $\left\langle\tilde{\pi}_{11}^{(0)}\right\rangle^{*}$, as evident from figure 2 .

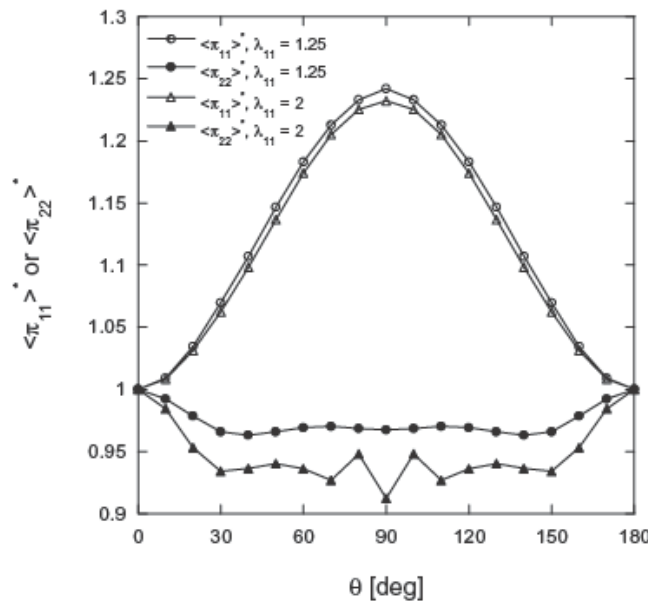

Figure 2. Evolution of the dimensionless stresses $\left\langle\tilde{\pi}_{11}^{(0)}\right\rangle^{*}$ and $\left\langle\tilde{\pi}_{22}^{(0)}\right\rangle^{*}$ as functions of the angle $\theta$ the REV is rotated from its initial configuration shown in figure 1(b). These stresses have been estimated for two imposed mesoscopic elongations $\lambda_{11}$.

- Whatever the considered elongation $\lambda_{11}$, figure 2 proves that the mesoscopic mechanical in-plane behaviour of the membrane exhibits orthotropy: stress ratios exhibit two symmetries along two orthogonal directions, i.e. for $\theta=0^{\circ}$ and $\theta=90^{\circ}$.

\subsection{Biaxial loadings}

We now return to the REV given in figure $1(\mathrm{~b})$ in order to analyse the effect of the mechanical loading type on its behaviour. For this reason the REV was here subjected to in plane and radial biaxial loadings corresponding to the following in plane mesoscopic transformation gradient:

$\underline{\tilde{\mathbf{F}}}^{(0)}=\lambda_{11}\left(\underline{\mathbf{e}}_{1} \otimes \underline{\mathbf{e}}_{1}+\beta \underline{\mathbf{e}}_{2} \otimes \underline{\mathbf{e}}_{2}\right)$

When it was possible, the elongation $\lambda_{11}$ along the $\mathrm{e}_{1}$ was increased until a value of 2 . The positive biaxial ratio $\beta=\lambda_{22} / \lambda_{11}$ was kept constant during each loading. Various numerical experiments were achieved with the following $\beta$ values : -1 (corresponding to an in-plane pure shear test), $-0.5,0$ (corresponding to a plane strain tension test), 0.5 and 1 (corresponding to an equibiaxial tension test).

Numerical results have been summarised in figure 3 , in which the stress-elongation diagrams $\left\langle\tilde{\pi}_{11}^{(0)}\right\rangle-\lambda_{11}$ and $\left\langle\tilde{\pi}_{22}^{(0)}\right\rangle-\lambda_{11}$ have been reported forthe different tested values of $\beta$, together with the deformed shapes of the REV at the end of 

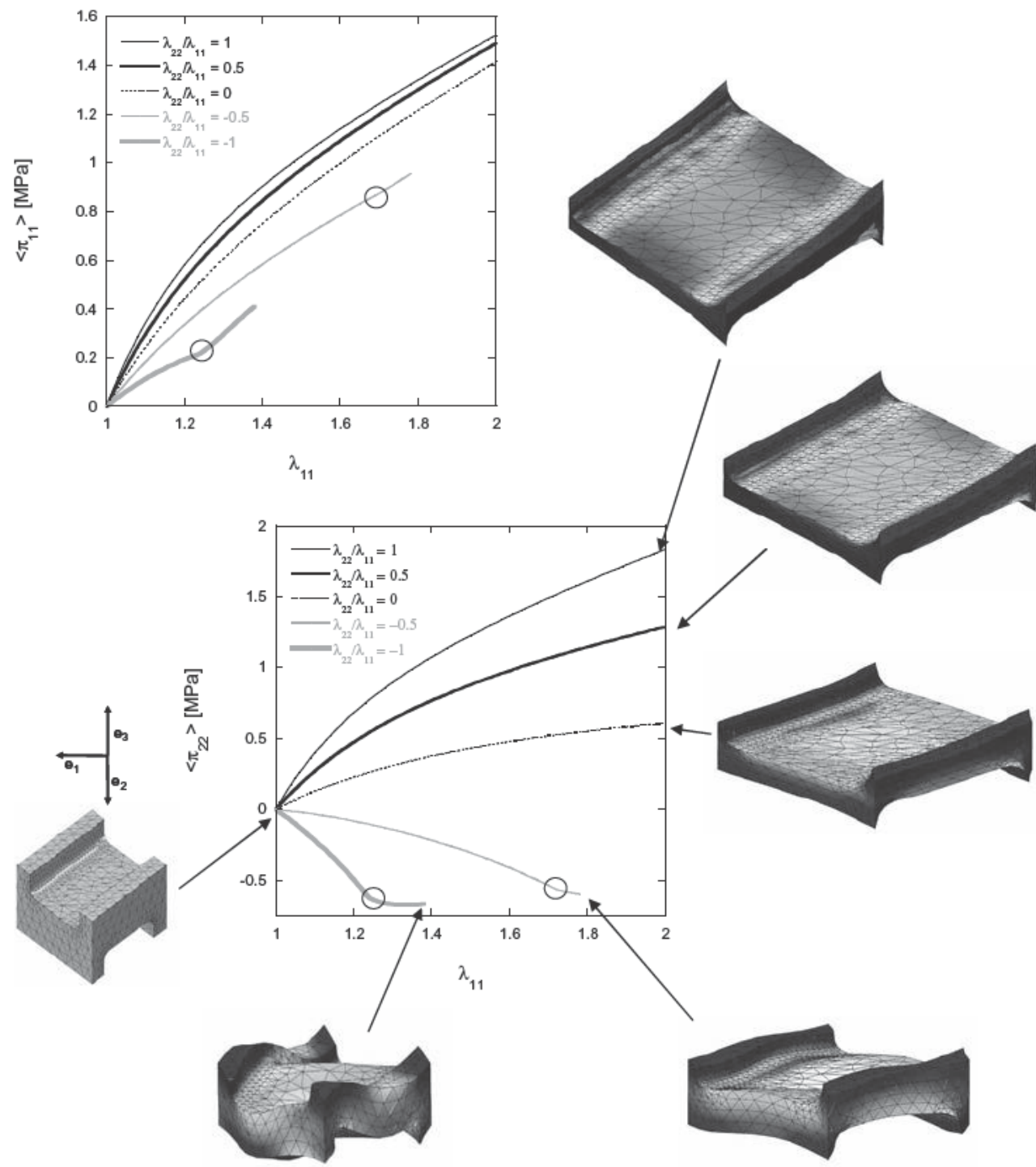

Figure 3. Biaxial loadings achieved with various elongation ratios $\beta=\lambda_{22} / \lambda_{11}$. The two graphs represent the evolution of themesoscopicstress components $\left\langle\tilde{\pi}_{11}^{(0)}\right\rangle$ (upper graph) and $\left\langle\tilde{\pi}_{22}^{(0)}\right\rangle$ (lower graph) as functions of the imposed mesoscopic elongation $\lambda_{11}$. We have also reported the initial mesh of the REV, as well as its deformed meshes at the end of the different mechanical loadings. Colormaps shown on the deformed meshed represent the values of the Von Mises stress, ranging from its lowest value (blue) to its maximal one (red).

the mechanical loadings. This figure brings up the following comments:

- The two stress-elongation graphs emphasise the key role of the mechanical loading on the mechanical response of the membrane. Whatever the considered stress component, an increase of $\beta$ yields to an increase of stress levels. Such a trend is much more pronounced with the stress component $\left\langle\tilde{\pi}_{22}^{(0)}\right\rangle$ than with $\left\langle\tilde{\pi}_{11}^{(0)}\right\rangle$.

- The anisotropy of the membrane, which was emphasised in the previous subsection, can also be observed when looking at stress-elongation curves in the case of the equibiaxial test, i.e. for $\beta=1$. Indeed, the two graphs of figure 3 , show 
that $\left\langle\tilde{\pi}_{22}^{(0)}\right\rangle$ is higher than $\left\langle\tilde{\pi}_{11}^{(0)}\right\rangle(20 \%$ higher when $\lambda_{11}=2$ ).

- Stress-elongation curves for $\beta \geq 0$ exhibit a curvature with a constant sign: this is directly due to the Neo-Hookean nature of the membrane material.

- Such an expected behaviour is not observed at all for stress elongation curves obtained with $\beta=-1$ and $\beta=-0.5$. Indeed, for these two mechanical loadings which involve compression stress components $\left\langle\tilde{\pi}_{22}^{(0)}\right\rangle$, the sign of the curvature suddenly change around $\lambda_{11}=1.25$ and $\lambda_{11}=1.75$ when $\beta=-1$ and $\beta=-0.5$, respectively (see the circles sketched in the graphs of figure 3 ). This change results in an anomalous increase of the strain hardening of $\left\langle\tilde{\pi}_{11}^{(0)}\right\rangle$ and in an anomalous strain softening of $\left\langle\tilde{\pi}_{22}^{(0)}\right\rangle$ By closely looking at the deformed shapes of the REV just after these transition zones (see figure 3 ), one can clearly see that the REV has been subjected to a local buckling along the $\underline{\mathbf{e}}_{2}$ direction, such a buckling being induced by the imposed meso-scopic compression elongation along this direction.

\section{CONCLUSIONS}

Within the framework proposed by the homogeni-sation method with multiple scale asymptotic expansions, and more precisely by pursuing the work of Pruchnicki (Pruchnicki 1998), we have proposed a method to analyse from numerical simulation the mesoscopic mechanical behaviour of heterogeneous hyperelastic membranes.

In particular, it has been shown that a membrane made up of a unique homogeneous material but with geometrical heterogeneities at the REV scale could exhibit significant anisotropy and possible microstructure instabilities such as buckling.
A deeper analysis of these phenomena as functions of both the membrane geometry (e.g. the inclination angle $\alpha$ ) and the imposed mesoscopic loading should be required.

The method also permits to analyse the mechanical behaviour under various mechanical loadings, thus allowing to constitute a precious and exhaustive database in order to propose relevant analytical forms of the mesoscopic law (14). This work is planned.

Before, its relevance must be proved. For that purpose, its prediction could be compared to what could be observed experimentally on similar heterogeneous membranes with a homogeneous hyperelastic material such as silicone rubber (Meunier et al. 2008). This work is also planned.

\section{REFERENCES}

Auriault, J.-L. (1991). Heterogeneous medium. is an equivalent macroscopic description possible? Int. J. Eng. Sci. 29(7), 785-795.

Bensoussan, A., J.-L. Lions, and G. Papanicolaou (1978). Asymptotic Analysis for Periodic Structures. Amsterdam: North Holland.

Caillerie, D. (1984). Thin elastic and periodic plates. Math. Meth. Appl. Sci. 6, 159-191.

Meunier, L. (2009). Etude Du Comportement Mécanique de Membranes de Silicone Á Anisotropie Contrôlée: Application À la Conception de Muscles Artificiels. Ph.D. thesis, Univ. de Grenoble, France.

Meunier, L., G. Chagnon, D. Favier, L. Orgéas, and P. Vacher (2008). Experimental and numerical study of the mechanical behaviour of an unfilled silicone rubber. Polym. Testing 27, 765-777.

Pruchnicki, E. (1998). Overal properties of thin hyperelastic plate at finite strain with edge effects using asymptotic method. Int. J. Eng. Sci. 36, 973-1000.

Sanchez-Palencia, E. (1980). Non-Homogeneous Media and Vibration Theory. Lecture Notes in Physics. Berlin, Germany: Springer-Verlag. 